\title{
Association of positively selected elF3a polymorphisms with toxicity of platinum-based chemotherapy in NSCLC patients
}

Ji-ye YIN ${ }^{1,2,3, *}$, Xiang-guang MENG ${ }^{4}$, Chen-yue QIAN ${ }^{1,2,3}$, Xiang-ping LI $^{5}$, Juan CHEN ${ }^{1,2,3}$, Yi ZHENG ${ }^{1,2,3}$, Rong LIU ${ }^{1,2,3}$, Hong-hao ZHOU ${ }^{1,2,3}$, Zhao-qian LIU $1,2,3, *$

\begin{abstract}
${ }^{1}$ Department of Clinical Pharmacology, Xiangya Hospital, Central South University, Changsha 410008, China; Institute of Clinical Pharmacology, Central South University; ${ }^{2}$ Hunan Key Laboratory of Pharmacogenetics, Changsha 410078, China; ${ }^{3}$ Hunan Province Cooperation Innovation Center for Molecular Target New Drug Study, Hengyang 421001, China; ${ }^{4}$ Laboratory of Cardiovascular Disease and Drug Research, Zhengzhou No 7 People's Hospital, Zhengzhou 450016, China; ${ }^{5}$ Department of Pharmacy, Xiangya Hospital, Central South University, Changsha 410008, China
\end{abstract}

Aim: Eukaryotic translation initiation factor 3 subunit A (elF3a) plays critical roles in regulating the initiation of protein translation, and elF3a is highly expressed in lung cancer. In this study, we investigated the association of the positively selected SNPs of elF3a with the response to and toxicity of platinum-based chemotherapy in Chinese patients with non-small cell lung cancer (NSCLC).

Methods: SNP data for elF3a locus were downloaded from HapMap database. For each SNP, haplotype, LD profile and population differentiation were analyzed. The long-range haplotype (LRH) test was employed to identify positively selected SNPs of elF3a. A total of 325 NSCLC patients were enrolled and genotyped for these SNPS.

Results: Five positively selected (rs1409314, rs4752219, rs4752220, rs7091672 and rs10510050) and 5 non-positively selected SNPs (rs10886342, rs11198804, rs2275112, rs10787899 and rs4752269) were identified in the LRH test. However, none of them was correlated with the platinum-based chemotherapy response. In contrast, 4 of the positively selected SNPs (rs1409314, rs4752219, rs4752220 and rs7091672) were significantly correlated with the toxicities tested (neutropenia, anemia, thrombocytopenia, emesis and hepatotoxicity). In addition, rs10510050 was significantly correlated with thrombocytopenia, emesis and hepatotoxicity. None of the 5 non-positively selected SNPs was correlated with the 5 toxicities.

Conclusion: The positively selected SNPs of elF3a are significantly correlated with platinum-based chemotherapy toxicities in Chinese NSCLC patients.

Keywords: elF3a; positive selection; polymorphism; lung cancer; platinum-based chemotherapy; chemotherapy toxicity; Chinese NSCLC patients

Acta Pharmacologica Sinica (2015) 36: 375-384; doi: 10.1038/aps.2014.160

\section{Introduction}

Translation is one of the major mechanisms of gene expression regulation. It consists of three major steps: initiation, elongation and termination. Among these, initiation is the rate-limiting step and involves at least 11 translation initiation factors $(\mathrm{eIFs})^{[1]}$. EIF3 is the largest and most complex, consisting of at least 13 subunits named eIF3a to eIF3m ${ }^{[2,3]}$. EIF3a is the largest subunit with a molecular weight of $170 \mathrm{kDa}$, and the gene

\footnotetext{
* To whom correspondence should be addressed.

E-mail liuzhaoqian63@126.com (Zhao-qian LIU); yinjiye2009@hotmail.com (Ji-ye YIN)

Received 2014-08-30 Accepted 2014-11-13
}

encoding it has 24 exons spanning approximately $45.8 \mathrm{~kb}$ of chromosome $10 \mathrm{q} 26^{[4]}$.

EIF3a plays critical roles in all steps of cap-dependent translation initiation, including binding with the $40 \mathrm{~S}$ ribosomal subunit, scanning and recognizing the start codon ${ }^{[2]}$. In addition, it has been reported to be involved in internal ribosomal entry site (IRES)-mediated cap-independent translation initiation $^{[5,6]}$. However, recent studies have shown that eIF3a is overexpressed in many cancers, including breast, lung, cervix, stomach and esophagus ${ }^{[2]}$. Our previous study also showed that eIF3a regulates drug response by altering the expression of some DNA repair proteins ${ }^{[7]}$. These studies thus implicate eIF3a as a potential oncogene. 
Single nucleotide polymorphisms (SNPs) are the most abundant human genome variations. Our previous study demonstrated that two eIF3a SNPs were correlated with drug response and toxicity ${ }^{[8,9]}$. We thus hypothesized that additional eIF3a SNPs may correlate with response and toxicity of platinum-based chemotherapy in non-small cell lung cancer (NSCLC) patients. It has been reported that screening SNPs with evidence of positive selection is a valid strategy for identifying functional SNPs ${ }^{[10-12]}$. This method is based on the theory that phenotypic differences between individuals are the functional consequences of genetic variants that facilitated the survival of ancestral human populations under different environmental stressors ${ }^{[13]}$. Therefore, SNPs under positive selection are likely to be functionally important for their linked genes. This strategy has been used successfully to identify functional SNPs associated with many genes, including glucose-6-phosphate dehydrogenase $(G 6 P D)^{[13]}$, multidrug resistance $1(M D R 1)^{[10]}$, cytochrome P450, family 3, subfamily A $(C Y P 3 A)^{[11]}$ and proprotein convertase subtilisin-like kexin type 9 (PCSK 9$)^{[12]}$.

In the present study, we employed the long-range haplotype (LRH) test to identify positively selected SNPs of eIF3a and explored whether they were correlated with response and toxicity of platinum-based chemotherapy in Chinese NSCLC patients.

\section{Materials and methods}

Study population, acquisition of clinical information and genotyping

The study protocol was approved by the Ethics Committee of Xiangya School of Medicine, Central South University. A total of 325 lung cancer patients were enrolled in this study. All individuals provided written informed consent in compliance with the code of ethics of the World Medical Association (Declaration of Helsinki) before this study was initiated. Eligible subjects were treated at the Affiliated Cancer Hospital or Xiangya Hospital of Central South University (Changsha, Hunan, China) between November 2011 and May 2013. For this study, patients had to meet the following criteria: (1) histologic or cytologic confirmation of lung cancer and (2) presence of a primary tumor. Exclusion criteria included (1) pregnancy or lactation, (2) active infection, (3) symptomatic brain or leptomeningeal metastases and (4) previous or concomitant other malignancies. All demographic and clinical information was obtained from the two hospitals. The clinical characteristics of all subjects are summarized in Table 1. We applied this study for clinical admission in the Chinese Clinical Trial Register (registration number: ChiCTR-RCC-12002830).

The chemotherapy response was evaluated by the response evaluation criteria in solid tumor (RECIST) guidelines. Complete response (CR) and partial response (PR) were defined as drug response, whereas stable disease (SD) and progressive disease (PD) were defined as drug resistance. In the current study, hematologic toxicity (anemia, neutropenia and thrombocytopenia) and gastrointestinal toxicity (emesis and hepatotoxicity) were evaluated. Toxicity was classified according to the National Cancer Institute Common Toxicity Criteria 3.0 (NCI-CTC 3.0) at each cycle of chemotherapy. For each patient, the most severe grade during the entire study interval was evaluated. In brief, for all side effects, grade 0 and grade $\geq 1$ were considered non-toxicity and toxicity, respectively. Genomic DNA of all subjects was isolated from the peripheral blood sample using a FlexiGene DNA Kit (Qiagen, Hilden, Germany) and stored at $4{ }^{\circ} \mathrm{C}$ until use. Genotyping was conducted by Sequenom's MassARRAY system (Sequenom, San Diego, California, USA).

\section{Polymorphism data and SNP marker selection}

Genotype data of SNPs were retrieved from the International HapMap Project database (Phase III Release \#28, www.hapmap.org). Six populations were included in this study, including CEU, CHB, JPT, YRI, ASW, and CHD. The ancestral allele of each SNP was defined by aligning the sequence of each SNP to a chimpanzee (Pan troglodyte) sequence from the UCSC database (genome.ucsc.edu).

SNPs spanning approximately $45.8 \mathrm{~kb}$ of the eIF3a locus were selected. To generate a more evenly distributed and denser SNP map, we extended the selection region to $200 \mathrm{~kb}$ upstream and downstream of the eIF3a locus. SNPs in the following categories were excluded from the study: (1) SNPs with a minor allele frequency $<0.05$, (2) SNPs that were not genotyped in at least one population and (3) SNPs departing from Hardy-Weinberg equilibrium. In total, 127 SNPs were included, and a flow chart describing SNP selection and information regarding included SNPs are provided in Figure S1 and Table S1, respectively.

\section{Data analysis}

Data analysis was performed using $\mathrm{R}$ and Bioconductor. In addition, the Haploview program was utilized to perform genetic analysis, including allele frequency description and selection, LD and haplotype block analysis ${ }^{[14]}$. Lewontin's coefficient $\left|D^{\prime}\right|$ and Pearson's correlation $r^{2[15]}$ were employed to estimate LD between SNP pairs. Statistical significance of LD between SNP pairs was analyzed by Arlequin ${ }^{[16]}$. The classical LD decay equation $D=D_{0}(1-\theta)^{t}$ was utilized to calculate LD decay trend line, which evaluated the overall LD in the populations ${ }^{[17]}$. Half-LD $\left(\mathrm{LD}_{0.5}\right)$ was defined as the distance at which $\left|D^{\prime}\right|=0.5$. Haplotype reconstruction was conducted in the PHASE program ${ }^{[18]}$. An unbiased estimator of $\mathrm{F}_{\mathrm{ST}}$ (F-statistic/fixation index) from pairwise population comparisons was calculated. Randomization method (1000 permutations) was used to test the statistical significance of $\mathrm{F}_{\mathrm{ST}}$ in the comparison using the FSTAT program ${ }^{[19]}$. A modified LRH test was used to assess for positive selection, and single SNP rather than haplotype was referred as "core" ${ }^{[13]}$. Haplotype homozygosity $(\mathrm{HH})$ was calculated in a stepwise manner as extended haplotype homozygosity $(\mathrm{EHH})^{[13]}$. Relative EHH (REHH) was the ratio of the EHH on the test core allele compared with the $\mathrm{EHH}$ of the grouped set of core alleles at the region not including the core allele tested ${ }^{[13]}$. Haplotype bifurcation diagram (HBD) was used to graphically evaluate the LD decay of both 
ancestral and derived alleles for a given core SNP. To test the statistical significance of the LRH test, an empirical distribution of core haplotype frequencies versus REHH was obtained by screening the entire chromosome 10 HapMap data in ASN, CEU and YRI populations. The HBD and LRH statistical tests were performed using Sweep software.

\section{Statistical analysis of association study}

All continuous variables were presented as means \pm SD and confidence intervals (95\% CI), and normally distributed data were analyzed by the two-sample $t$ test. Noncontiguous variables, genotype distributions, and allelic frequencies in different groups were compared using the $x^{2}$ test. Unconditional logistic regression was performed to estimate the association of drug response and toxicity with eIF3a polymorphisms by calculating odds ratios (OR) and their $95 \%$ confidence intervals (CI). The $P$ value was two-sided and $P<0.05$ was considered statistically significant. The aforementioned statistical analyses were performed using PLINK ${ }^{[20]}$ and SPSS 18.0 software (SPSS Inc, Chicago, Illinois, USA). Bonferroni correction was used to correct multiple testing.

\section{Results}

\section{Haplotype and LD profile}

We first detected the haplotype and LD profile of the involved SNPs, and the pair-wise $\left|D^{\prime}\right|$ and $r^{2}$ were calculated to determine the IIF3a LD pattern. As shown in Figure 1, the patterns of LD structure were highly different across the entire locus between Africans and non-Africans. There are 18, 16, 17, 14, 29, and 29 blocks observed in CHD, CHB, JPT, CEU, ASW, and
YRI populations, respectively. The longest blocks spanned 91 $\mathrm{kb}$ in CHD, $91 \mathrm{~kb}$ in CHB, $91 \mathrm{~kb}$ in JPT, $94 \mathrm{~kb}$ in CEU, 48 kb in ASW, and $26 \mathrm{~kb}$ in YRI.

We then plotted the $\left|D^{\prime}\right|$ and $r^{2}$ between SNP pairs against their physical distance, as shown in Figure 2. The LD generally decreased with physical distance. A curve was then fitted to determine the half $\mathrm{LD}\left(\mathrm{LD}_{50}\right)$ in all populations. The genetically older populations were expected to have lower LDs, and this was the case in our study. The $L_{50} \mathrm{~s}$ of African populations YRI (44.5 kb) and ASW (61.5 kb) were shorter than those of non-African populations, while the longest $\mathrm{LD}_{50} \mathrm{~s}$ presented in the Chinese population were CHD (78.3 kb) and CHB (75.4 $\mathrm{kb})$.

Furthermore, we calculated the proportion of SNP pairs with significant LD in these populations and observed clear differences. As shown in Figure S2A, African populations had fewer SNP pairs with significant LDs: the proportions were $23.66 \%$ and $19.03 \%$ in YRI and ASW populations. For nonAfrican populations, however, 39.07\%, 38.72\%, 36.71\%, and $35.88 \%$ SNP pairs showed significant LD in the CHD, CEU, $\mathrm{CHB}$, and JPT populations, respectively. We also plotted the proportion of SNP pairs with insignificant LDs against physical distance for ASN (East Asian population; combined CHB and JPT populations), CEU and YRI populations (Figure S2B). In accordance with previous observation, the African YRI population showed lower LD levels, while the ASN population showed higher allelic association.

Together, the haplotype and LD pattern displayed high diversity among the 6 populations analyzed, especially between African (ASW and YRI) and non-African (CHD, CHB,
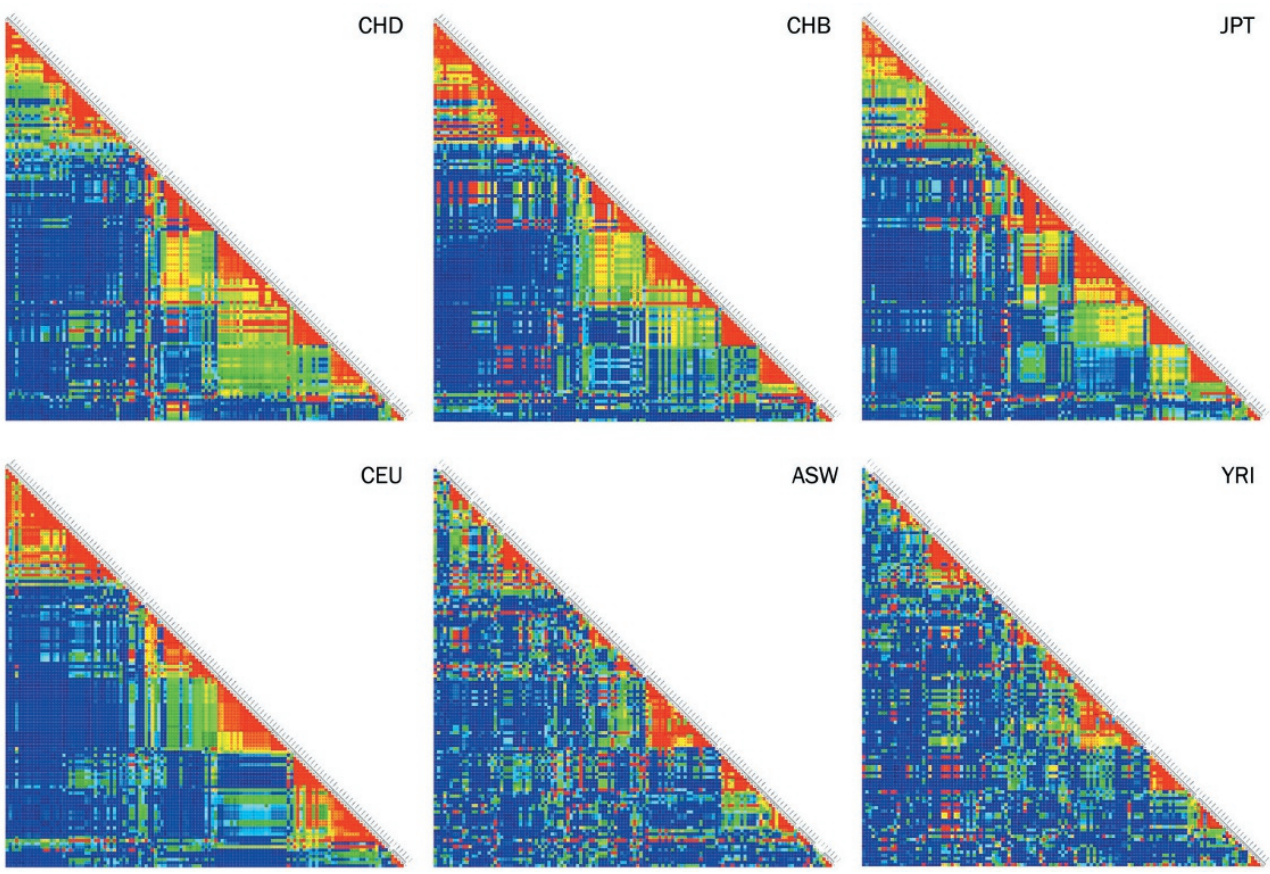

Figure 1. LD pattern of elF3a polymorphisms in the CHD, CHB, JPT, CEU, ASW, and YRI populations. Pairwise LD between polymorphisms was measured by $\left|D^{\prime}\right|$. Haplotype blocks partitioned in each population were also numbered above them. The analysis was performed in the Haploview program (version 4.2). 
A
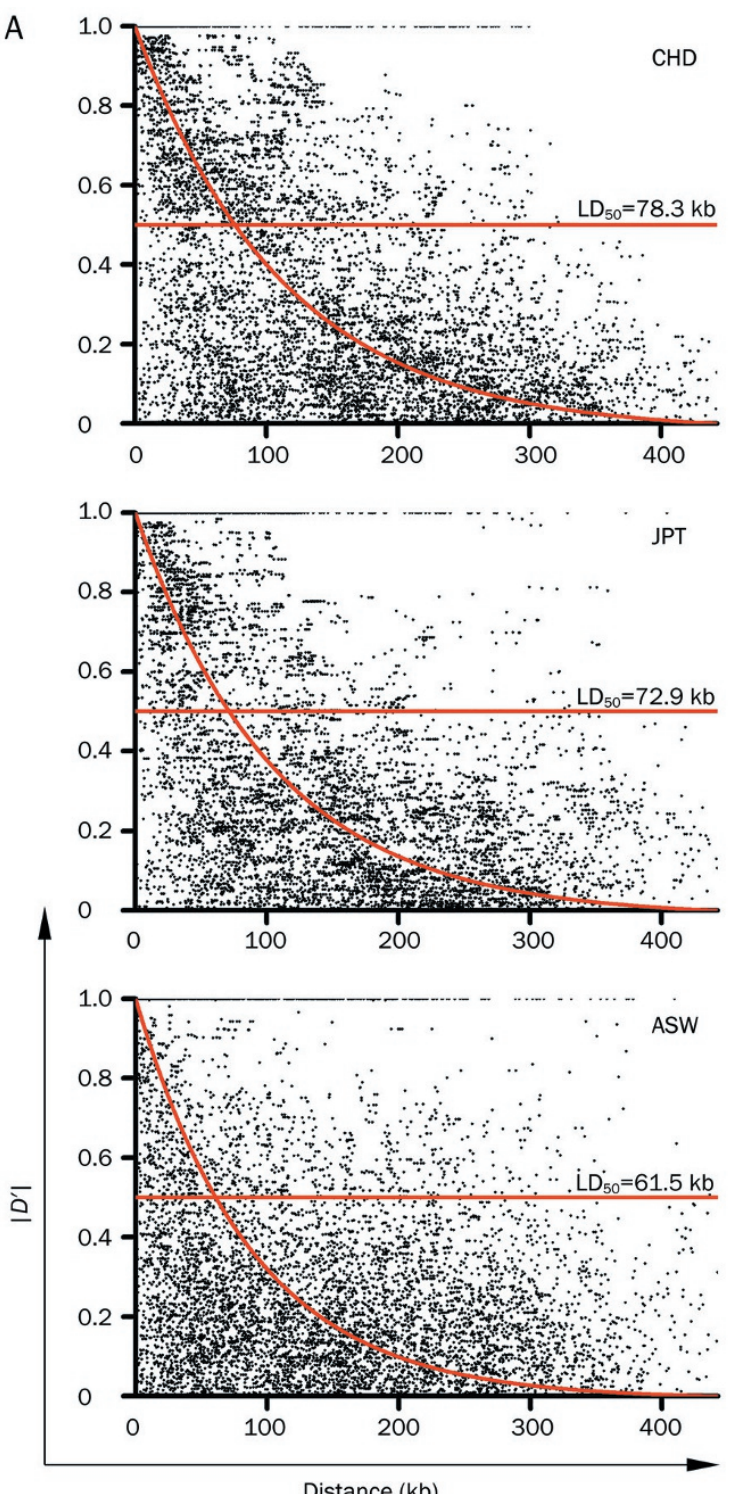


Figure 2A. LD decay profile of elF3a polymorphisms in the CHD, CHB, JPT, CEU, ASW, and YRI populations. (A) $\left|D^{\prime}\right|$ between elF3a SNP pairs were calculated and plotted against their physical distance in the CHD, CHB, JPT, CEU, ASW, and YRI populations. A classical LD decay equation was utilized to calculate LD decay trend line, which is indicated by a red line in the figure. The LD $_{50}$ was also shown. Details of the calculation were described in the Materials and methods section.

CEU, and JPT) populations.

\section{Population differentiation}

To further test for differences in $e I F 3 a$ allelic frequency among populations, we calculated $\mathrm{F}_{\mathrm{ST}}$ values. Considering that the $\mathrm{CHB}$ and JPT populations exist in the same region and have substantial similarity in their genetic makeup, they were combined to make one ASN population in further analyses. Additionally, the following analyses were conducted on only the ASN, CEU, and YRI populations, which reflected a substantial amount of the genetic variation found throughout the world $^{[21]}$.

Based on the pairwise $\mathrm{F}_{\mathrm{ST}}$ values, eIF3a showed high popula- tion differentiation. SNPs with a proportion of $21.71 \%$ in CEU versus ASN, $43.41 \%$ in CEU versus YRI and $33.33 \%$ in ASN versus YRI showed significantly high $\mathrm{F}_{\mathrm{ST}}$, respectively. We next plotted the $\mathrm{F}_{\mathrm{ST}}$ values against physical distance in Figure S3. As shown, 7 SNPs in CEU versus YRI and 5 SNPs in ASN versus YRI had $\mathrm{F}_{\mathrm{ST}}$ values above the threshold of 0.45 , which was considered the threshold value of selection pressure ${ }^{[22,23]}$. However, none of the SNPs in CEU versus ASN had $\mathrm{F}_{\mathrm{ST}}$ values above 0.45 , indicating that eIF3a had low levels of differentiation between these two populations.

These results indicated that there was significantly different population differentiation among the ASN, CEU, and YRI populations, and that some SNPs may be under 
B
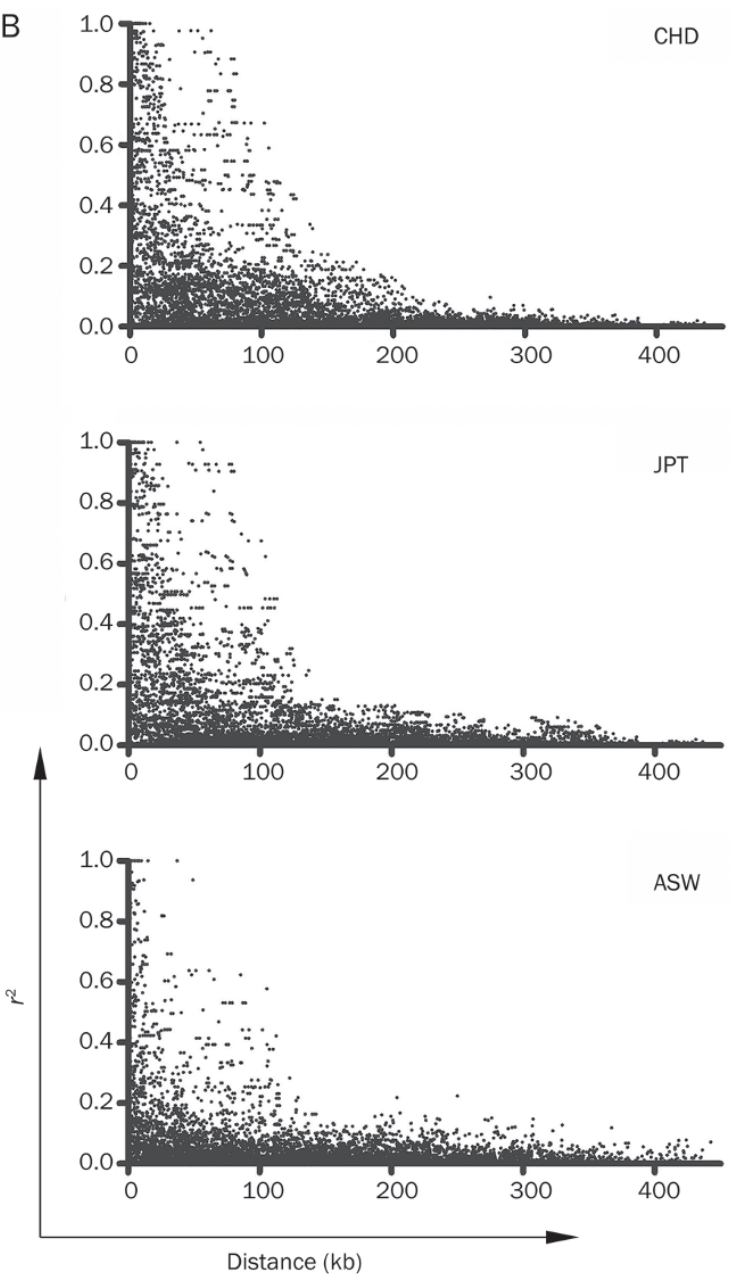
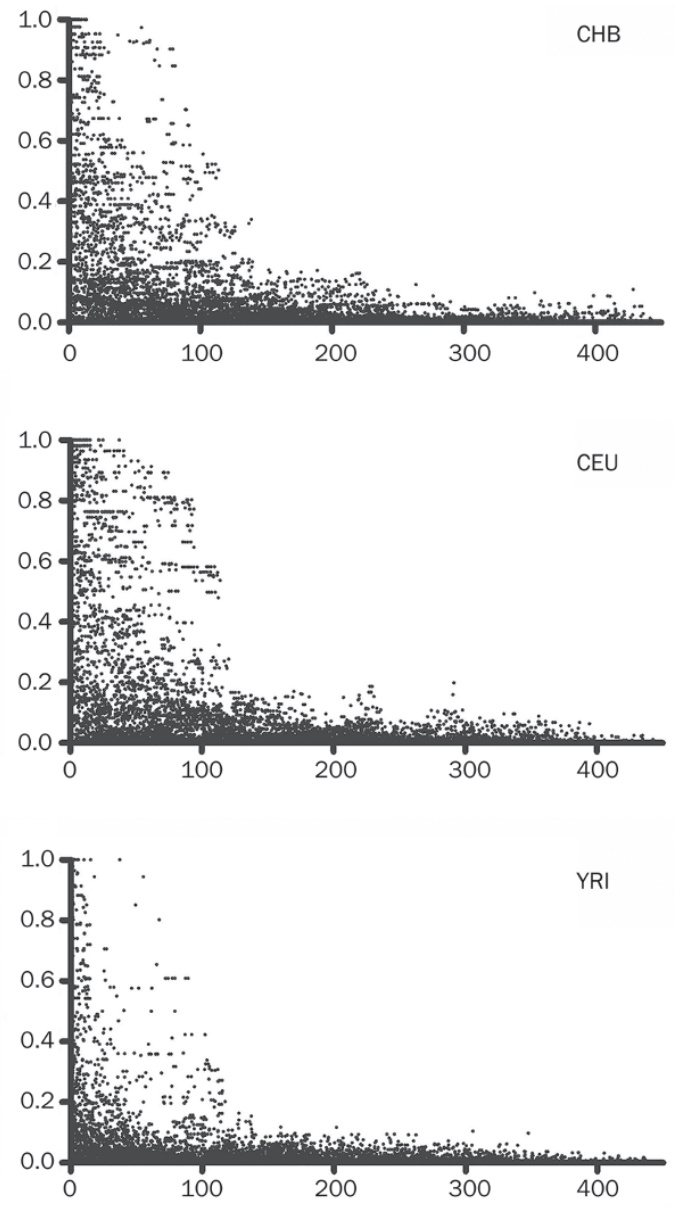

Figure 2B. LD decay profile of elF3a polymorphisms in the CHD, CHB, JPT, CEU, ASW, and YRI populations. (B) $r^{2}$ between elF3a SNP pairs were calculated and plotted against their physical distance in the CHD, CHB, JPT, CEU, ASW, and YRI populations. Details of the calculation were described in the Materials and methods section.

selective pressure.

\section{Signature of positive selection of elF3a}

To test for evidence of positive selection occurring at these SNPs with eIF3a, we performed an LRH test. We first plotted the relative EHH (REHH) of core SNPs against their allele frequencies. Based on the empirical distribution, 41, 33, and 13 SNPs showed significant departure from evolutionary neutrality in the YRI, ASN, and CEU populations, respectively. The top 5 SNPs with lowest $P$ values were then involved in further study. As shown in Figure 3, A allele of SNP rs1409314 $(P=0.0012), C$ allele of SNP rs4752219 $(P=0.0025)$, A allele of SNP rs4752220 $(P=0.0025)$, T allele of SNP rs7091672 $(P=0.0043)$ and A allele of SNP rs10510050 $(P=0.0046)$ deviated significantly from evolutionary neutrality. To assess this result, the EHH decay of these 5 SNPs was plotted against the physical distance. Generally, the overall EHH of the rs1409314A, rs4752219C, rs4752220A, rs7091672T, and rs10510050A alleles decayed more slowly than their corresponding ancestral/ derived alleles in all three populations (Figure S4). To confirm this result, we plotted haplotype branching diagrams (HBD) for these 5 SNPs, as indicated in Figure S5. Consistent with previous results, the rs1409314A, rs4752219C, rs4752220A, rs7091672T, and rs10510050A alleles had obvious long-range LD across all populations, especially in YRI, as indicated by a single distinctly predominant thick branch. Taken these results together, we concluded that rs1409314, rs4752219, rs4752220, rs7091672, and rs10510050 showed a signature of recent positive selection.

As a control, we further analyzed 5 randomly selected SNPs (rs10886342, rs11198804, rs2275112, rs10787899, and rs4752269), which didn't show evidence of positive selection. These 5 SNPs were selected as representative non-positively selected SNPs of eIF3a for use in the following study.

\section{Association of selected SNPs and platinum-based chemotherapy} response

Our previous study demonstrated that eIF3a may act as an 

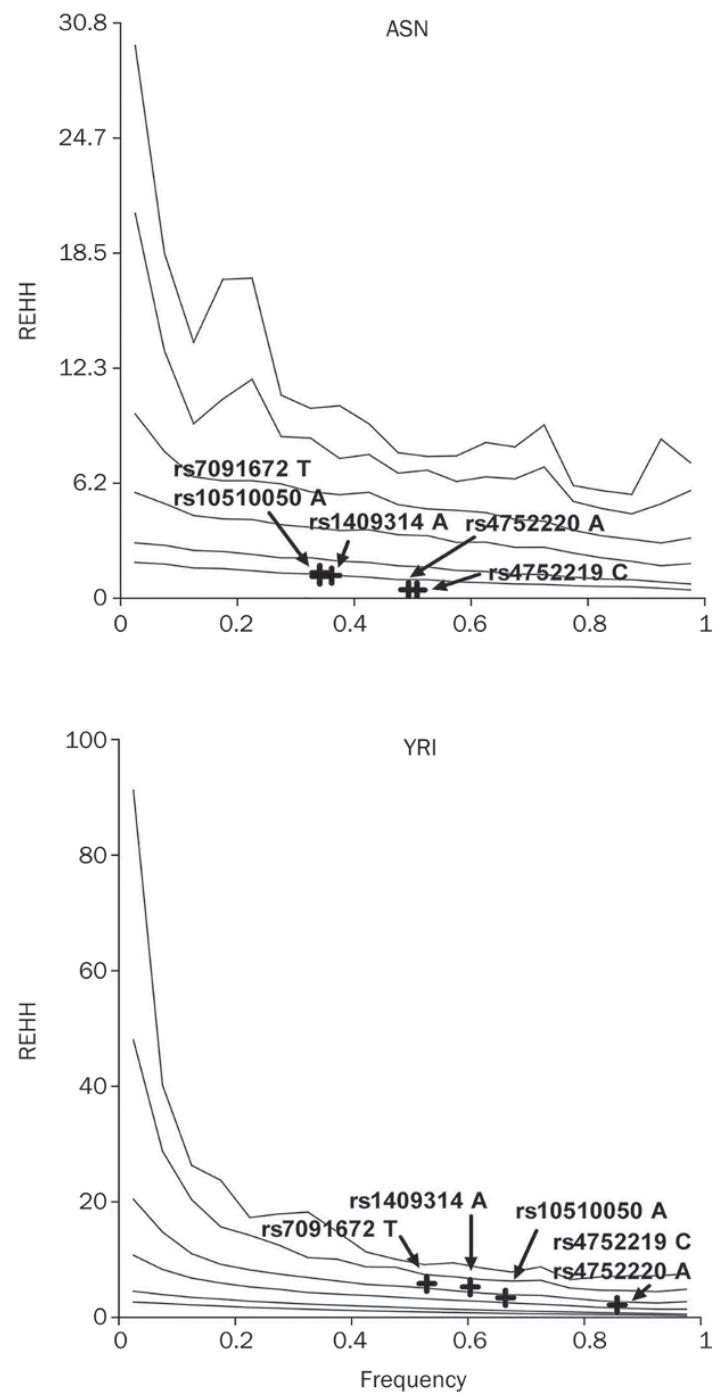

oncogene and may be correlated with lung cancer chemotherapy response and toxicity ${ }^{[4,6,7]}$. Thus, we tested whether these positively selected SNPs were associated with platinumbased chemotherapy response in Chinese NSCLC patients. A total of 325 lung cancer patients were recruited for genotyping of the aforementioned 5 positively selected and 5 nonpositively selected SNPs. There were significant differences between responders and non-responders in terms of age and chemotherapy regimen, suggesting that these factors need to be adjusted in the statistical analysis (Table 1). All tested SNPs were consistent with Hardy-Weinberg equilibrium in both responders and non-responders.

Table 2 summarizes the results of association study of 10 SNPs in additive, dominant and recessive models. As indicated, neither the 5 positively selected SNPs (rs1409314, rs4752219, rs4752220, rs7091672 and rs10510050) nor the 5 nonpositively selected SNPs (rs10886342, rs11198804, rs2275112, rs10787899, and rs4752269) were significantly associated with NSCLC patients' platinum-based chemotherapy response.

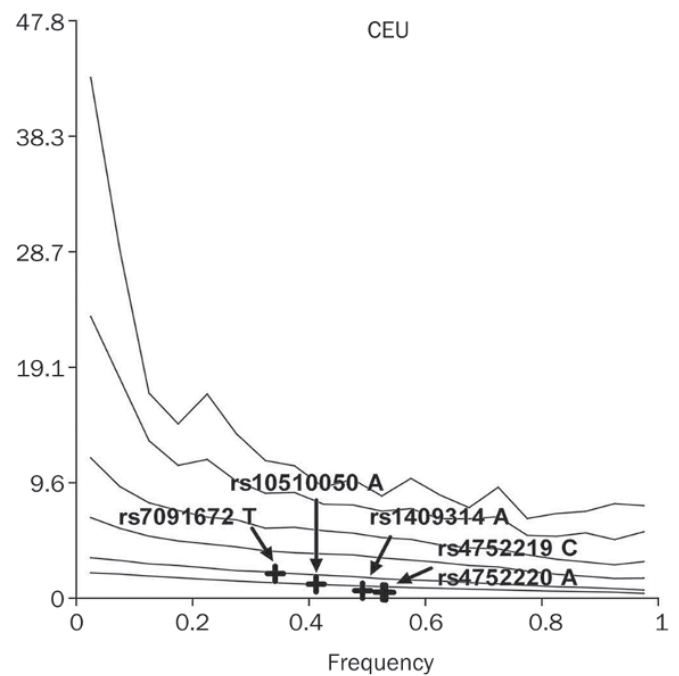

Figure 3. LRH test for 5 positively selected SNPs. The REHH value of 5 core alleles was plotted against its frequency in the ASN, CEU and YRI populations. The points of REHH against allele frequency were based on the calculation of chromosome 10 phased data downloaded from the HapMap database (data point not shown in the figure). The 50th, 75th, 90th, 95th, 99th, and 99.9th percentile lines were obtained by binning the empirical data by allele frequencies into 20 bins with equal intervals of 0.05 . All analysis was performed in the Sweep programe.

\section{Association of selected SNPs and platinum-based chemotherapy toxicity}

We next tested whether positively selected SNPs were correlated with chemotherapy toxicity in these patients. In the present study, we investigated 5 toxicities: neutropenia, anemia, thrombocytopenia, emesis and hepatotoxicity. As indicated in Table 3, each was divided into two groups: toxicity (0 grade) and non-toxicity ( $\geq 1$ grade). In general, $64 \%-68 \%$ patients showed at least one toxicity and only 32\%-36\% patients did not suffer from any toxicity.

It is interesting to note that 4 (rs1409314, rs4752219, rs4752220, and rs7091672) of the 5 positively selected SNPs were significantly correlated with all tested toxicities (Table 4). In addition, a significant association was also detected between rs10510050 and thrombocytopenia $(\mathrm{OR}=4.34,95 \% \mathrm{CI}=1.47-$ 12.81, $P=0.00)$, emesis $(\mathrm{OR}=0.39,95 \% \mathrm{CI}=0.18-0.83, P=0.01)$ and hepatotoxicity $(\mathrm{OR}=0.58,95 \% \mathrm{CI}=0.34-0.99, P=0.04)$. In contrast, of the 5 non-positively selected SNPs (rs10886342, rs11198804, rs2275112, rs10787899, and rs4752269), none 
Table 1. Baseline characteristic of NSCLC patients treated with platinum-based chemotherapy.

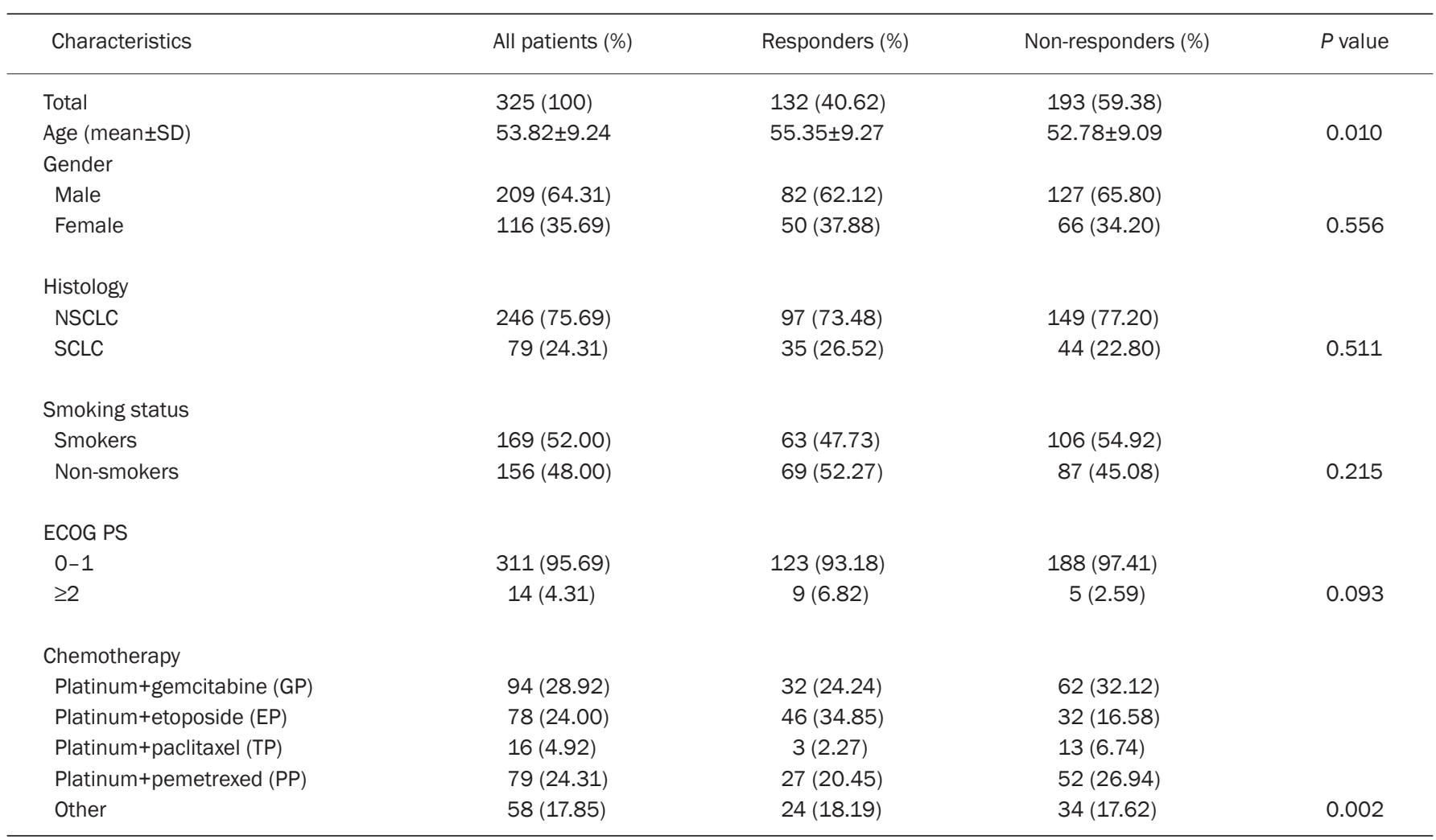

showed significant association with any of the 5 tested toxicities. These results together suggest that the aforementioned 5 positively selected eIF3a SNPs are significantly correlated with platinum-based chemotherapy toxicities in Chinese NSCLC patients.

\section{Discussion}

In the present study, we first characterized the haplotype and LD profile of eIF3a in data obtained from the HapMap database, then analyzed the population differentiation in three populations (ASN, CEU, and YRI). Moreover, we identified 5 SNPs (rs1409314, rs4752219, rs4752220, rs7091672, and rs10510050) of eIF3 $a$ that had undergone recent positive selection using the LRH test. Finally, we demonstrated that these SNPs were correlated with platinum-based chemotherapy toxicities in Chinese NSCLC patients.

With the explosion of newly identified SNPs, it is becoming impractical to investigate every SNP individually for correlation with a disease or drug response. Although new methods of DNA sequencing and genotyping have been developed, these are time consuming and costly. Thus, bioinformatics methods have been developed to identify potential functional SNPs. Based on the theories of natural selection and the aforementioned "Out-of-Africa" hypothesis, searching for signatures of recent positive selection can be used to identify potential functional SNPs ${ }^{[2]}$. With the dispersal of humans from Africa to the other regions of the world, different popu- lations faced various selective pressures presented by climate, pathogens and sources of food. Thus, functional SNPs that facilitated survival in ancestral human populations may result in phenotypic differences among modern individuals or groups ${ }^{[25]}$. These SNPs may be retained under the pressure of positive selection; for example, a classic G6PD-202A variant that confers malaria resistance showed a strong evidence of recent positive selection ${ }^{[13]}$. Other groups have successfully utilized this method to detect functional SNPs in PCSK9 ${ }^{[12]}$ and $A B C$ drug transporter genes ${ }^{[26]}$. In the current study, we employed this method to identify 5 positively selected eIF3a SNPs and confirmed that they were significantly correlated with platinum-based chemotherapy toxicities in Chinese NSCLC patients. Importantly, another 5 non-positively selected control SNPs did not show evidence of association. Although our previous studies identified a number of platinum-based chemotherapy responses and toxicities associated $\mathrm{SNPS}^{[27-29]}$, this time- and cost-saving method is a potentially powerful tool for specifically identifying SNPs that correlate with drug response in the future.

Although eIF3a is the largest subunit of eIF3, which is itself the largest and most complex initiation factor, it has been shown to be dispensable for the function of eIF3-medicated translation initiation ${ }^{[2,30]}$. By contrast, previous studies by our group and others have suggested that eIF3a is a potential oncogene. Thus, it is important to understand the contributions of eIF3a polymorphisms to its correlation with cancers. 
Table 2. Association of elF3a SNPs with platinum-based chemotherapy response in the Chinese patients.

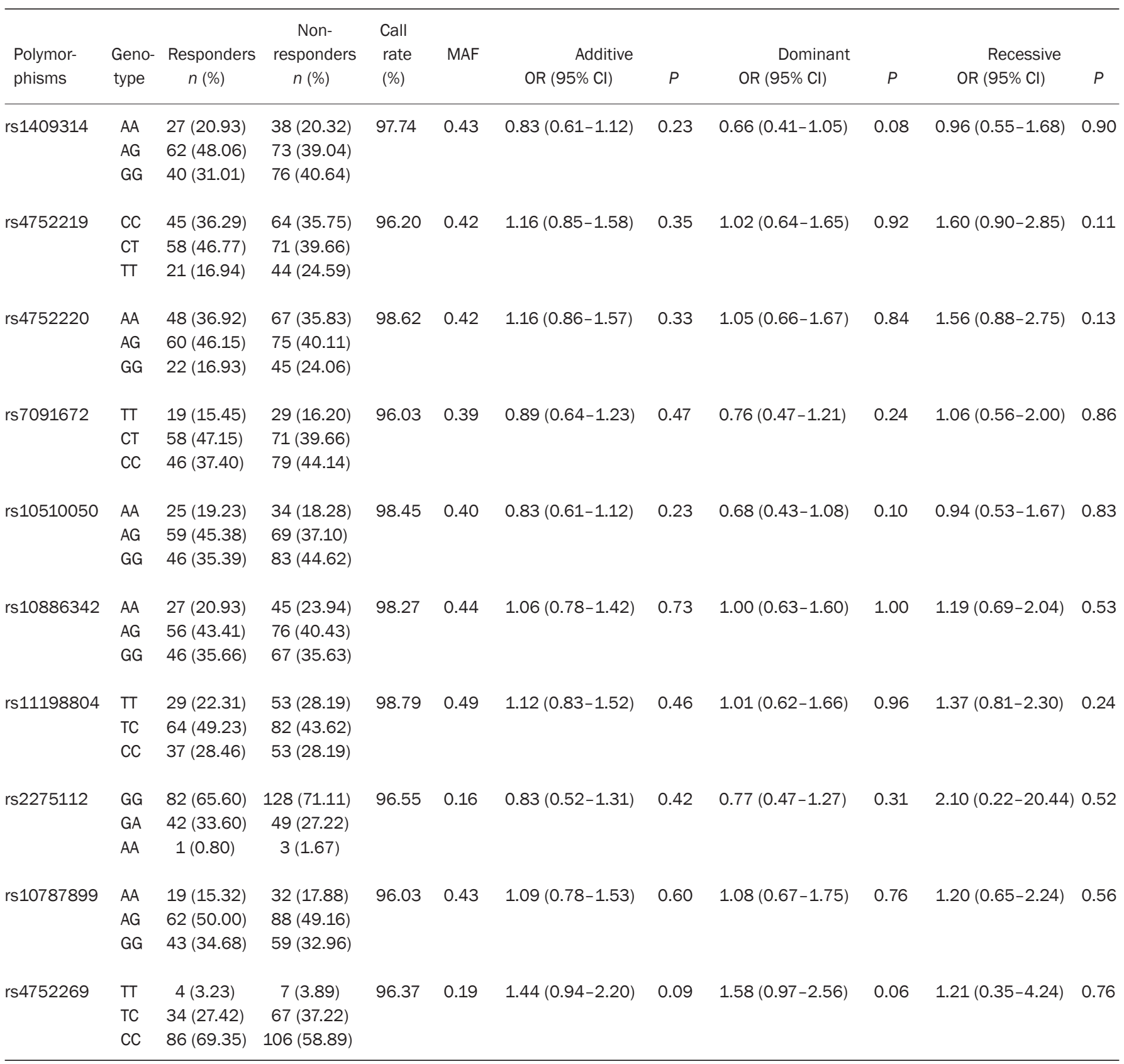

Table 3. Groups of graded platinum-based chemotherapy toxicities.

\begin{tabular}{lll}
\hline \multicolumn{1}{c}{ Side effect } & 0 grade $n(\%)$ & $\geq 1$ grade $n(\%)$ \\
\hline Neutropenia & $112(34.46)$ & $213(65.54)$ \\
Anemia & $114(35.08)$ & $211(64.92)$ \\
Thrombocytopenia & $114(35.08)$ & $211(64.92)$ \\
Emesis & $116(35.69)$ & $209(64.31)$ \\
Hepatotoxicity & $104(32.00)$ & $221(68.00)$ \\
\hline
\end{tabular}

Our previous study investigated the correlation of eIF3a polymorphisms and platinum-based chemotherapy resistance and severe toxicity in the Chinese population ${ }^{[8,9]}$. Olson et al found that eIF3a rs10787899 was significantly correlated with breast cancer risk in Caucasian female populations ${ }^{[31]}$. In the current study, we further identified 5 polymorphisms which correlated with lung cancer platinum-based chemotherapy toxicities in the Chinese population. While the mechanisms of the correlation remain unknown, we speculate that these 
Table 4. Association of elF3a SNPs with platinum-based chemotherapy toxicities in the Chinese patients. ${ }^{\mathrm{b}} P<0.05$.

\begin{tabular}{|c|c|c|c|c|c|c|c|c|c|c|}
\hline \multirow{2}{*}{ SNPs } & \multicolumn{2}{|l|}{ Leukopenia } & \multicolumn{2}{|l|}{ Anemia } & \multicolumn{2}{|c|}{ Thrombocytopenia } & \multicolumn{2}{|l|}{ Emesis } & \multicolumn{2}{|l|}{ Hepatotoxicity } \\
\hline & OR (95\% Cl) & $P$ & OR $(95 \% \mathrm{Cl})$ & $P$ & OR $(95 \% \mathrm{Cl})$ & $P$ & OR (95\% Cl) & $P$ & OR $(95 \% \mathrm{Cl})$ & $P$ \\
\hline rs1409314 & $2.36(1.23-4.53)$ & $0.01^{\mathrm{b}}$ & $2.44(1.27-4.67)$ & $0.01^{b}$ & $2.44(1.27-4.67)$ & $0.01^{\mathrm{b}}$ & $2.47(1.29-4.74)$ & $0.01^{\mathrm{b}}$ & $1.58(1.14-2.61)$ & $0.01^{b}$ \\
\hline rs4752219 & $1.64(1.12-2.39)$ & $0.01^{b}$ & $1.58(1.09-2.30)$ & $0.02^{b}$ & $1.64(1.07-2.75)$ & $0.02^{b}$ & $1.55(1.07-2.25)$ & $0.02^{b}$ & $1.52(1.02-2.53)$ & $0.02^{b}$ \\
\hline rs4752220 & $1.64(1.12-2.39)$ & $0.01^{b}$ & $1.58(1.09-2.30)$ & $0.02^{b}$ & $1.64(1.07-2.75)$ & $0.02^{b}$ & $1.55(1.07-2.25)$ & $0.02^{b}$ & $1.52(1.02-2.53)$ & $0.02^{b}$ \\
\hline rs7091672 & $3.44(1.32-8.98)$ & $0.01^{b}$ & $2.84(1.16-6.92)$ & $0.02^{b}$ & $1.60(1.05-2.68)$ & $0.02^{b}$ & $2.88(1.18-7.02)$ & $0.02^{b}$ & $1.64(1.07-2.75)$ & $0.02^{b}$ \\
\hline rs10510050 & $1.01(0.47-2.21)$ & 0.97 & $0.78(0.36-1.69)$ & 0.52 & $4.34(1.47-12.81)$ & $0.00^{\mathrm{b}}$ & $0.39(0.18-0.83)$ & $0.01^{\mathrm{b}}$ & $0.58(0.34-0.99)$ & $0.04^{b}$ \\
\hline rs10886342 & $1.13(0.53-2.42)$ & 0.75 & $1.31(0.63-2.74)$ & 0.46 & $0.51(0.16-1.65)$ & 0.25 & $0.89(0.48-1.66)$ & 0.72 & $0.95(0.59-1.53)$ & 0.84 \\
\hline rs11198804 & $1.42(0.66-3.06)$ & 0.36 & $1.21(0.58-2.53)$ & 0.61 & $1.05(0.37-3.04)$ & 0.92 & $1.40(0.77-2.57)$ & 0.27 & $1.06(0.66-1.69)$ & 0.81 \\
\hline rs2275112 & $1.24(0.46-3.37)$ & 0.67 & $0.85(0.29-2.52)$ & 0.76 & $2.11(0.65-6.87)$ & 0.21 & $0.91(0.48-1.82)$ & 0.85 & $1.27(0.69-2.34)$ & 0.44 \\
\hline rs10787899 & $0.50(0.21-1.21)$ & 0.12 & $1.06(0.49-2.27)$ & 0.89 & $0.78(0.26-2.34)$ & 0.65 & $1.26(0.90-1.77)$ & 0.18 & $1.39(0.87-2.25)$ & 0.17 \\
\hline rs4752269 & $1.48(0.61-3.61)$ & 0.39 & $0.85(0.32-2.29)$ & 0.75 & $2.24(0.74-6.81)$ & 0.14 & $0.68(0.28-1.66)$ & 0.39 & $0.84(0.46-1.56)$ & 0.59 \\
\hline
\end{tabular}

SNPs may be potential genetic markers for drug response and toxicity.

In the present investigation, we extended the SNP selection region to $200 \mathrm{~kb}$ upstream and downstream of the eIF3a locus to generate a more even distribution. However, some regions selected overlapped with other genes, including family with sequence similarity 45, member A (FAM45A) in the eIF3a 5' untranslated region (5' UTR) and nanos homolog 1 (Drosophila) (NANOS1) in the eIF3a 3' UTR regions. Indeed, the 5 positively selected SNPs (rs1409314, rs4752219, rs4752220, rs7091672, and rs10510050) were located in the overlap region of NANOS1 and eIF3a. Thus, they may be considered genetic markers for both genes. This point is especially important for in vitro functional study, where both genes should be considered in the investigation. It should be noted that some limitations need to be considered when interpreting results of this study. First, although we confirmed the function of these positively selected SNPs in clinical patients, we did not conduct a study in vitro. Therefore, how these SNPs affected eIF3a function still remains unknown. Another limitation came from our sample size. The current study had a relatively small sample size and lacked confirmation from other validation stage populations. Additionally, we only detected the correlation in the Chinese population. Thus, this result needs to be further confirmed in a larger sample size and in different ethnic populations.

In summary, we identified 5 positively selected eIF3a SNPs using the LRH test and confirmed that they were significantly correlated with platinum-based chemotherapy toxicities in Chinese NSCLC patients. Our results imply that this strategy of SNP selection may be an inexpensive, effective way to discovery of SNPs correlated to drug response and toxicities.

\section{Acknowledgements}

This work was supported by National High-tech R\&D Program of China (863 Program Grant, No 2012 AA02A517 and 2012AA02A518), National Natural Science Foundation of China (№ 81173129, 81202595, 81373490, and 81273595), and Hunan Provincial Natural Science Foundation of China (№ 2015JJ1024).

\section{Author contribution}

Ji-ye YIN, Hong-hao ZHOU, and Zhao-qian LIU conceived and designed the experiments. Ji-ye YIN, Xiang-guang MENG, Juan CHEN, and Yi ZHENG performed the experiments. Ji-ye YIN, Xiang-guang MENG, and Rong LIU analyzed the data. Chen-yue QIAN and Xiang-ping LI contributed reagents, materials and analysis tools. Ji-ye YIN and Zhao-qian LIU wrote the paper.

\section{Supplementary Information}

Supplementary Tables and Figures are available on the Acta Pharmacologica Sinica's website.

\section{References}

1 Sonenberg N, Hershey JWB, Mathews MB. Translational control of gene expression. Cold Spring Harbor (NY): Cold Spring Harbor Laboratory Press; 2000.

2 Yin JY, Dong Z, Liu ZQ, Zhang JT. Translational control gone awry: a new mechanism of tumorigenesis and novel targets of cancer treatments. Biosci Rep 2011; 31: 1-15.

3 Dong Z, Zhang JT. Initiation factor elF3 and regulation of mRNA translation, cell growth, and cancer. Crit Rev Oncol Hematol 2006; 59: 169-80.

4 Pincheira R, Chen Q, Zhang JT. Identification of a 170-kDa protein over-expressed in lung cancers. Br J Cancer 2001; 84: 1520-7.

5 Buratti E, Tisminetzky S, Zotti M, Baralle FE. Functional analysis of the interaction between HCV 5'UTR and putative subunits of eukaryotic translation initiation factor elF3. Nucleic Acids Res 1998; 26: 3179-87.

6 Yin JY, Dong ZZ, Liu RY, Chen J, Liu ZQ, Zhang JT. Translational regulation of RPA2 via internal ribosomal entry site and by elF3a. Carcinogenesis 2013; 34: 1224-31.

7 Yin JY, Shen J, Dong ZZ, Huang Q, Zhong MZ, Feng DY, et al. Effect of elF3a on response of lung cancer patients to platinum-based chemotherapy by regulating DNA repair. Clin Cancer Res 2011; 17: 4600-9.

8 Xu X, Han L, Duan L, Zhao Y, Yang H, Zhou B, et al. Association between elF3alpha polymorphism and severe toxicity caused by platinum-based chemotherapy in non-small cell lung cancer patients. Br J Clin Pharmacol 2013; 75: 516-23.

9 Xu X, Han L, Yang H, Duan L, Zhou B, Zhao Y, et al. The A/G allele of elF3a rs3740556 predicts platinum-based chemotherapy resistance 
in lung cancer patients. Lung Cancer 2013; 79: 65-72.

10 Tang K, Wong LP, Lee EJ, Chong SS, Lee CG. Genomic evidence for recent positive selection at the human MDR1 gene locus. Hum Mol Genet 2004; 13: 783-97.

11 Chen X, Wang H, Zhou G, Zhang X, Dong X, Zhi L, et al. Molecular population genetics of human CYP3A locus: signatures of positive selection and implications for evolutionary environmental medicine. Environ Health Perspect 2009; 117: 1541-8.

12 Ding K, Kullo IJ. Molecular population genetics of PCSK9: a signature of recent positive selection. Pharmacogenet Genomics 2008; 18 : 169-79.

13 Sabeti PC, Reich DE, Higgins JM, Levine HZ, Richter DJ, Schaffner SF, et al. Detecting recent positive selection in the human genome from haplotype structure. Nature 2002; 419: 832-7.

14 Barrett JC, Fry B, Maller J, Daly MJ. Haploview: analysis and visualization of LD and haplotype maps. Bioinformatics 2005; 21 : 263-5.

15 Lewontin RC. Annotation: the analysis of variance and the analysis of causes. Am J Hum Genet 1974; 26: 400-11.

16 Excoffier L, Lischer HE. Arlequin suite ver 3.5: a new series of programs to perform population genetics analyses under Linux and Windows. Mol Ecol Resour 2010; 10: 564-7.

17 Schulze TG, Chen YS, Akula N, Hennessy K, Badner JA, McInnis MG, et al. Can long-range microsatellite data be used to predict short-range linkage disequilibrium. Hum Mol Genet 2002; 11: 1363-72.

18 Stephens M, Smith NJ, Donnelly P. A new statistical method for haplotype reconstruction from population data. Am J Hum Genet 2001; 68: 978-89.

19 JG FSTAT, a program to estimate and test gene diversities and fixation indices (version2.9.3). Available from http://www.unil.ch/izea/softwares/fstat.html. Updated from Goudet (1995). 2001.

20 Purcell S, Neale B, Todd-Brown K, Thomas L, Ferreira MA, Bender D, et al. PLINK: a tool set for whole-genome association and population- based linkage analyses. Am J Hum Genet 2007; 81: 559-75.

21 International HapMap Consortium. The International HapMap Project. Nature 2003; 426: 789-96.

22 Akey JM, Zhang G, Zhang K, Jin L, Shriver MD. Interrogating a highdensity SNP map for signatures of natural selection. Genome Res 2002; 12: 1805-14.

23 Rosenberg NA, Pritchard JK, Weber JL, Cann HM, Kidd KK, Zhivotovsky LA, et al. Genetic structure of human populations. Science 2002; 298: 2381-5.

24 Biswas S, Akey JM. Genomic insights into positive selection. Trends Genet 2006; 22: 437-46.

25 Bamshad M, Wooding SP. Signatures of natural selection in the human genome. Nat Rev Genet 2003; 4: 99-111.

26 Wang Z, Wang J, Tantoso E, Wang B, Tai AY, Ooi LL, et al. Signatures of recent positive selection at the ATP-binding cassette drug transporter superfamily gene loci. Hum Mol Genet 2007; 16: 1367-80.

27 Li XP, Yin JY, Wang Y, He H, Li X, Gong WJ, et al. The ATP7B genetic polymorphisms predict clinical outcome to platinum-based chemotherapy in lung cancer patients. Tumour Biol 2014; 35: 8259-65.

28 Wang Y, Yin JY, Li XP, Chen J, Qian CY, Zheng Y, et al. The association of transporter genes polymorphisms and lung cancer chemotherapy response. PLoS One 2014; 9: e91967.

29 Wang Y, Li XP, Yin JY, Zhang Y, He H, Qian CY, et al. Association of HMGB1 and HMGB2 genetic polymorphisms with lung cancer chemotherapy response. Clin Exp Pharmacol Physiol 2014; 41: 40815.

30 Chaudhuri J, Chakrabarti A, Maitra U. Biochemical characterization of mammalian translation initiation factor 3 (elF3). Molecular cloning reveals that $\mathrm{p} 110$ subunit is the mammalian homologue of Saccharomyces cerevisiae protein Prt1. J Biol Chem 1997; 272: 30975-83.

31 Olson JE, Wang X, Goode EL, Pankratz VS, Fredericksen ZS, Vierkant $\mathrm{RA}$, et al. Variation in genes required for normal mitosis and risk of breast cancer. Breast Cancer Res Treat 2010; 119: 423-30. 